\title{
Oleanolic acid attenuates renal fibrosis in mice with unilateral ureteral obstruction via facilitating nuclear translocation of Nrf2
}

Sungjin Chung ${ }^{1,2}$, Hye Eun Yoon ${ }^{1,3}$, Soo Jeong Kim¹, Sung Jun Kim ${ }^{1,3}$, Eun Sil Koh ${ }^{1,2}$, Yu Ah Hong ${ }^{5}$, Cheol Whee Park ${ }^{1,4}$, Yoon Sik Chang ${ }^{1,2}$ and Seok Joon Shin ${ }^{1,3^{*}}$

\begin{abstract}
Background: Renal interstitial fibrosis is a common final pathological process in the progression of kidney disease. This is primarily due to oxidative stress, which contributes to renal inflammation and fibrosis. Nuclear factor-erythroid-2-related factor 2 (Nrf2) is known to coordinate induction of genes that encode antioxidant enzymes. We investigated the effects of oleanolic acid, a known Nrf2 activator, on oxidative stress-induced renal inflammation and fibrosis.

Methods: One day before unilateral ureteral obstruction (UUO) performed in C57BL/6 mice, oleanolic acid treatment was initiated and was continued until 3 and 7 days after UUO. Renal inflammation and fibrosis, markers of oxidative stress, and changes in Nrf2 expression were subsequently evaluated.

Results: In the obstructed kidneys of UUO mice, oleanolic acid significantly attenuated UUO-induced collagen deposition and fibrosis on day 7. Additionally, significantly less inflammatory cell infiltration, a lower ratio of Bax to Bcl-2 expression, and fewer apoptotic cells on TUNEL staining were observed in the obstructed kidneys of oleanolic acid-treated mice. Oleanolic acid increased the expression of nuclear Nrf2, heme oxygenase-1, NAD(P)H:quinone oxidoreductase 1 and heat shock protein 70, and decreased lipid peroxidation in the obstructed kidney of UUO mice. There were no changes in the expression of total Nrf2 and Kelch-like ECH-associated protein 1, indicating that oleanolic acid enhanced nuclear translocation of Nrf2.
\end{abstract}

Conclusions: These results suggest that oleanolic acid may exert beneficial effects on renal fibrosis by increasing nuclear translocation of Nrf2 and subsequently reducing renal oxidative stress.

\section{Background}

Tubulointerstitial fibrosis is the final common pathological feature in the progression to end-stage renal disease irrespective of the type of primary glomerular injury, such as hypertensive nephrosclerosis, diabetic nephropathy or glomerulonephritis $[1,2]$. Over the last few years, numerous studies have been performed to identify the pathogenesis of renal fibrosis, and there has been increasing evidence that oxidative stress is an important factor in its progression. Reactive oxygen species (ROS) are produced in response to various insults to the kidney, and

\footnotetext{
* Correspondence: imkidney@catholic.ac.kr

'Department of Internal Medicine, College of Medicine, The Catholic University of Korea, 222 Banpo-daero, Seoul 137-701, Republic of Korea ${ }^{3}$ Division of Nephrology, The Catholic University of Korea Incheon St. Mary's Hospital, 56, Dongsu-ro, Bupyeong-gu, Incheon 403-720, Republic of Korea Full list of author information is available at the end of the article
}

there are several reports that oxidative stress plays a significant role in renal damage in obstructed kidneys [3-5]. ROS cause tubulointerstitial injury by lipid peroxidation, increasing hydrogen peroxides, DNA breakdown, and protein damage [6]. ROS has also been found to play an important role in kidney fibrosis by regulation of inflammatory monocyte and macrophage infiltration, proliferation of interstitial fibroblasts, and extracellular matrix accumulation in the renal interstitium [6,7].

Oleanolic acid is a natural triterpenoid which has recently attracted considerable attention for its antioxidant properties. These properties have been tested in experimental models of drug-induced hepatotoxicity, multiple sclerosis, hypertension, atherosclerosis, and lung injury [8-14]. According to previous studies, the induction of nuclear factor-erythroid-2-related factor 2 (Nrf2) activation may mediate this oleanolic acid-induced antioxidant 
effect $[8,15]$. Nrf2 is a transcription factor that is widely known to be protective against oxidative stress and damage [16].

To gain further insight into the mechanisms that modulate renal fibrosis, we investigated whether up-regulation of Nrf2-dependent antioxidative signaling ameliorates renal inflammation and fibrosis. We used oleanolic acid, which is an Nrf2 activator, as the antioxidant in a mouse model of renal fibrosis induced by unilateral ureteral obstruction (UUO).

\section{Methods}

\section{Animal preparation}

All animal studies were conducted with the approval of The Institutional Animal Care and Use Committee sevenor eight-week-old male C57BL/6 mice weighting 20-25 g (OrientBio, Inc., Seoul, Republic of Korea) were used in this study. Animals were housed in standard cages in a room with constant temperature on a 12-hour light-dark cycle. They were fed a standard pellet laboratory chow (OrientBio, Inc., Seoul, Republic of Korea) and had free access to water. Oleanolic acid was dissolved in $2 \% \mathrm{w} / \mathrm{v}$ dimethyl sulfoxide (DMSO) and then diluted with distilled water for each injection to a final DMSO concentration of $0.2 \% \mathrm{w} / \mathrm{v}$. Therefore, the vehicle used was $0.2 \% \mathrm{w} / \mathrm{v}$ DMSO. The dose of oleanolic acid was chosen based on a previous report [10]. Oleanolic acid or vehicle was administered intraperitoneally one day before UUO, and was continued for 3 or 7 days after surgery. UUO or sham surgery was performed as described previously [17]. In the UUO group, the left ureter was exposed through a midabdominal incision and ligated using 4-0 silk under general anesthesia. Sham-operated mice had their ureters exposed and manipulated without ligation. Mice were divided into a total of five groups as follows: sham, UUOcontrol day 3, UUO-oleanolic acid (OA) day 3, UUOcontrol day 7 and UUO-OA day $7(\mathrm{n}=8$, each group). Groups of mice were sacrificed at 3 and 7 days after the operation, and the obstructed kidneys were removed for tissue analysis.

\section{Quantitative determination of tissue collagen content}

The total collagen content in kidney tissue was measured by acid hydrolysis of the kidney tissue section as described previously [18]. Briefly, each kidney sample was weighed, hydrolysed in $6 \mathrm{~N} \mathrm{HCl}$ for 18 hours at $110^{\circ} \mathrm{C}$, and dried thoroughly at $75^{\circ} \mathrm{C}$. Dried samples were solubilized in citric acid collagen buffer $(0.23 \mathrm{~mol} / \mathrm{L}$ citric acid, $0.88 \mathrm{~mol} / \mathrm{L}$ sodium acetate trihydrate, $0.85 \mathrm{~mol} / \mathrm{L}$ sodium hydroxide, and $1.2 \%$ acetic acid) and filtered through a $0.45 \mu \mathrm{m}$ centrifugal filter unit (Ultrafree-MC, Millipore, Billerica, MA, USA). Samples were diluted in citric acid collagen buffer and then loaded into each well of a microplate, to which $100 \mu \mathrm{L}$ of fresh chloramine-T solution $(1.4 \%$ chloramine-T and $10 \%$ n-propanol in citric acid buffer) was added to start the oxidation reaction. The microplate was incubated for $15 \mathrm{~min}$ at room temperature, and $100 \mu \mathrm{L}$ of Ehrlich's reagent (15\% 4-dimethylamino-benzaldehyde, 62\% n-propanol, and $18 \%$ perchloric acid) was added to each well to start the colour reaction. The microplate was incubated in a large water bath for $20 \mathrm{~min}$ at $65^{\circ} \mathrm{C}$. The amount of hydroxyproline was measured by a spectrophotometric assay at $550 \mathrm{~nm}$. Total collagen in the kidney tissue was calculated on the assumption that collagen contains $12.7 \%$ hydroxyproline by weight.

\section{Histologic examination}

Histologic analyses were performed using paraffin-embedded tissues. To evaluate the severity of tubular injury and tubulointerstitial fibrosis, kidney sections were processed and stained with hematoxylin and eosin (H\&E) and Masson trichrome, respectively. Renal tubular injury was assessed and given a score from 0 to $3(0=$ normal, $1=$ slight damage, $2=$ moderate damage, $3=$ severe damage) as previously described [19]. Fibrotic area was quantified using MetaMorph imaging software (Molecular Devices Inc., Downingtown, PA, USA) in more than 10 randomly selected fields from cortex and medulla. The ratio of the fibrotic area to the total selected field was indicated as the severity of tubulointerstitial fibrosis. Immunohistochemistry for F4/80 and TdT-mediated dUTP nick end labelling (TUNEL) were performed. After deparaffinization, the sections were hydrated, incubated in $0.5 \%$ Triton X-100PBS solution for 30 minutes, and washed three times with PBS. Nonspecific binding sites were blocked with normal donkey serum diluted 1:10 in PBS for 1 hour, and the sections were incubated overnight in a humidified chamber at $4^{\circ} \mathrm{C}$ with primary antibody raised against F4/80 (rat antimouse F4/80 monoclonal antibody, Abcam, Cambridge, MA, USA). After rinsing in PBS, the sections were incubated in peroxidase-conjugated anti-mouse IgG (Jackson ImmunoResearch Laboratories, West Grove, PA, USA) for 1 hour. For coloration, the sections were incubated with a mixture of $0.05 \%$ 3,3'-diaminobenzidine containing $0.01 \%$ $\mathrm{H}_{2} \mathrm{O}_{2}$ at room temperature until a brown color was visible, and were then washed with Tris buffer ( $\mathrm{pH} 7.6)$, counterstained with hematoxylin, and observed under light microscopy (Zeiss LSM 510, Carl Zeiss, Jena, Germany). Twenty high-power fields that included the renal cortex and corticomedullary junction were randomly selected in each section, and proportional areas of staining were quantified using MetaMorph imaging software (Molecular Devices Inc., Downingtown, PA, USA). Apoptotic cells were identified using a TUNEL assay. This was performed on paraffin sections fixed with $4 \%$ paraformaldehyde according to the manufacturer's instructions (Millipore, Billerica, MA, USA). TUNEL-positive apoptotic cells were counted in 20 randomly selected tubulointerstitial areas of 
each kidney sample. All slides were analyzed in a blinded fashion.

\section{Western blot analysis}

The total protein content of the renal tissues was extracted using a Pro-Prep Protein Extraction Kit (iNtRON Biotechnology, Inc., Seongnam, Gyeonggi-do, Republic of Korea) according to the manufacturer's instructions. The protein concentration was determined using a BCA protein assay (Pierce Biotechnology, Rockford, IL, USA). Equal kidney protein samples were separated by $15 \%$ SDS-PAGE and transferred to nitrocellulose membranes. Nuclear extracts, which were used for Nrf2 immunoblotting, were also prepared with the NE-PER nuclear kit as previously described (Pierce Biotechnology, Rockford, IL) [8]. For immunodetection, the blots were incubated overnight in PBS containing 0.1\% Tween-20 and 5\% skim milk with primary antibodies raised against the following proteins: heme oxygenase (HO)-1 (diluted 1:200, BD Biosciences, San Jose, CA, USA), heat shock protein 70 (Hsp70) (1:1000, ENZO Life Sciences, Inc., Farmingdale, NY, USA), NAD(P)H:quinone oxidoreductase 1 (NQO1) (1:500, Santa Cruz Biotechnology, Santa Cruz, CA, USA), MnSOD (1:2000, Abcam, Cambridge, MA, USA), catalase (1:2000; Abcam), Bax (1:500, Santa Cruz Biotechnology), Bcl-2 (1:100, Santa Cruz Biotechnology), Kelch-like ECHassociated protein 1 (Keap1; 1:1000; Abcam), and Nrf2 (1:1000; Abcam). The blots were washed and incubated with a secondary antibody conjugated with horseradish peroxidase (Jackson ImmunoResearch Laboratories, West Grove, PA, USA), and the protein bands were visualized using the chemiluminescence detection system (ImageQuant LAS 4000 mini, GE Healthcare, Piscataway, NJ, USA). Intensity values were determined using Image-Pro Plus software (Media Cybernetics, Bethesda, MD, USA).

\section{Measurement of oxidative stress}

To determine the level of lipid peroxidation in the kidney tissues, the level of malondialdehyde (MDA), a biomarker of free radical-mediated oxidative stress, was measured as previously described [20]. Briefly, the kidney tissue was homogenized in sucrose buffer $(70 \mathrm{mM}$ sucrose, $210 \mathrm{mM}$ mannitol, $1 \mathrm{mM}$ EDTA, $10 \mathrm{mM}$ Hepes in distilled water). After protein quantification, $100 \mu \mathrm{g}$ of kidney lysates were mixed with $1 \mathrm{ml}$ of thiobarbituric acid-trichloroacetic acid- $\mathrm{HCl}$ solution $(0.375 \%$ thiobarbituric acid, trichloroacetic acid in $0.25 \mathrm{~N} \mathrm{HCl}, \mathrm{pH} 2.0$ ) and boiled at $100^{\circ} \mathrm{C}$ for 15 minutes. Absorbance was measured at a wavelength of $535 \mathrm{~nm}$. The hydrogen peroxide $\left(\mathrm{H}_{2} \mathrm{O}_{2}\right)$ levels in the kidney tissues were determined using Fox reagent $(0.25 \mathrm{M} \mathrm{H} 2 \mathrm{SO} 4,1 \mathrm{M}$ sorbitol, $25 \mathrm{mM}$ ferrous ammonium sulfate, and $1 \mathrm{mM}$ xylenol orange in distilled water) as previously described [20]. $\mathrm{H}_{2} \mathrm{O}_{2}$ oxidizes iron (II) to iron (III) in the presence of sorbitol, which acts as a catalyst for the reaction. Iron (III) then forms a purple complex with xylenol orange. Absorbance was measured at a wavelength of $560 \mathrm{~nm}$.

\section{Statistical analysis}

Data are expressed as means \pm SE. Statistical differences among groups were calculated using ANOVAs with Bonferroni correction. P-values of $<0.05$ were considered to be statistically significant.

\section{Results}

Tubular injury, interstitial fibrosis, total collagen content and interstitial inflammatory cell infiltration in renal tissue

Tubular injury in the obstructed kidney was markedly increased after UUO (Figure 1A). Oleanolic acid significantly decreased tubular injury in the obstructed kidney on both day 3 and day 7 (Figure 1B). The severity of renal fibrosis also increased after UUO in a time-dependent manner, as seen in Masson trichrome staining (Figure 1A). The degree of tubulointerstitial fibrosis tended to be lower in the UUO-OA mice compared to those of UUO-control mice on day 3. However, this difference was not statistically significant. In contrast, the tubulointerstitial fibrotic areas in the obstructed kidneys from UUO-OA prominently decreased compared to those from UUO-control mice on day 7 (Figure 1C).

Immunohistochemistry for $\mathrm{F} 4 / 80$ was performed to investigate the degree of interstitial inflammatory cell infiltration. The number of F4/80-positive cells increased according to time course of UUO (Figure 1A). Oleanolic acid markedly decreased renal F4/80-positive cells infiltration in the obstructed kidneys from UUO-OA mice on both day 3 and day 7 (Figure 1D).

The amount of total collagen in the obstructed kidneys was found to be increased after UUO. Oleanolic acid significantly decreased the total collagen content in the obstructed kidneys from UUO-OA mice compared to that from UUO-control mice on day 7 (Figure 2).

\section{Keap1 and Nrf2 expressions}

The levels of intra-renal Keap1 and Nrf2 were determined using western blot analysis (Figure 3A). Under basal conditions, Nrf2 is located in the cytoplasm as an inactive complex bound to a repressor molecule, Keap1 [8]. Various stimuli including oxidants and antioxidants result in dissociation of Nrf2 from Keap1 and its translocation to the nucleus [16]. The expression of Keap1 was not changed after UUO, and oleanolic acid did not change the level of Keap1 in UUO mice on both day 3 and day 7 (Figure 3B). The expression of total Nrf2 showed a pattern similar to that of Keap1 (Figure 3A). On the contrary, oleanolic acid markedly increased the levels of nuclear Nrf2 in the obstructed kidneys. Therefore, nuclear Nrf2/ 


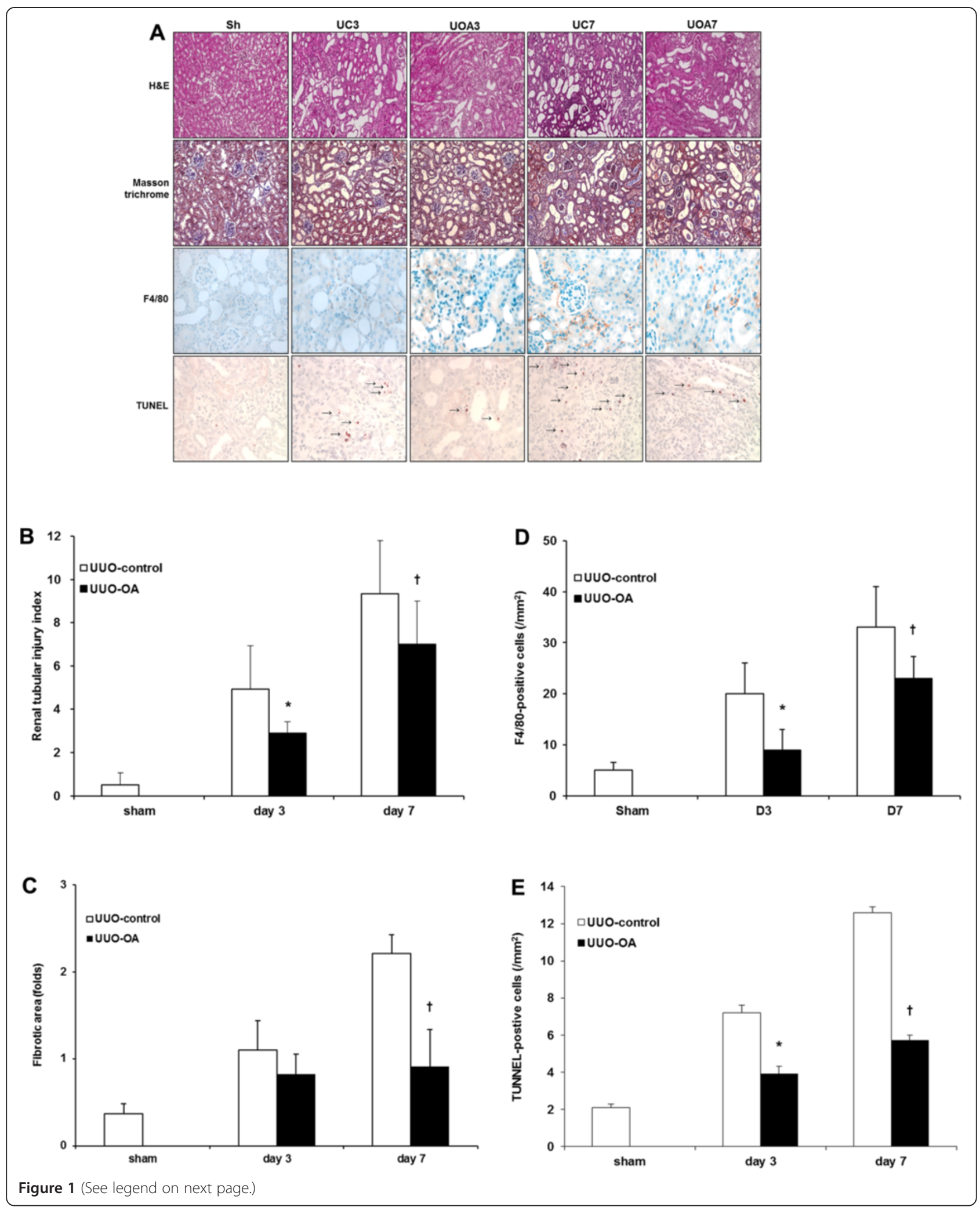


(See figure on previous page.)

Figure 1 Effects of oleanolic acid (OA) on renal morphological changes in unilateral ureteral obstruction (UUO). (A) Representative photographs assessing renal tubular injury (H\&E, x200), interstitial fibrosis (Masson trichrome, x200), immunohistochemical staining for the infiltration of F4/80-positive cells (x400) and TUNEL staining (x400). Black arrows indicate TUNEL-positive cells (brown color). (B) Renal tubular injury index. (C) Semiquantitative analysis of interstitial fibrosis. (D) Number of F4/80-positive cells. (E) Number of TUNEL-positive cells. Sh, sham; UC3, UUO-control day 3; UOA3, UUO-oleanolic acid day 3; UC7, UUO-control day 7; UOA7, UUO-oleanolic acid day 7; *P < 0.05 versus UUO-control day 3 group; ${ }^{\dagger} P<0.05$ versus UUO-control day 7 group.

total Nrf2 ratio was significantly increased in the UUOOA mice compared with those of UUO-control mice on both days 3 and day 7 (Figure 3C).

\section{Renal oxidative stress}

Oxidative stress induced by UUO was assessed by measuring the expression of HO-1, Hsp70, NQO1, catalase, and MnSOD using western blot analysis (Figure 4A), as well as the renal tissue levels of $\mathrm{H}_{2} \mathrm{O}_{2}$ and lipid peroxidation. The expression of $\mathrm{HO}-1$ showed increased levels after UUO according to time dependent manner. It was significantly greater in the obstructed kidneys of UUO-OA mice compared to that of UUO-control mice on both day 3 and day 7 (Figure 4B). The expression of Hsp70 showed no significant differences between sham and UUO-control mice. Oleanolic acid did not significantly increase the expression of Hsp70 on day 3. However, it markedly increased the level of Hsp70 on day 7 (Figure 4C). The expression of NQO1 was decreased after UUO; however, oleanolic acid significantly increased the level of NQO1 on day 7 (Figure 4D). On the other hand, the expression of catalase, an $\mathrm{H}_{2} \mathrm{O}_{2}$-inducible anti-oxidant enzyme, was not different between groups, and oleanolic acid showed no effect on the levels of catalase (Figure 4E). The expressions of MnSOD in experimental groups showed similar patterns to those of catalase (Figure 4F).

Direct measurement of the tissue levels of $\mathrm{H}_{2} \mathrm{O}_{2}$ and lipid peroxidation in the obstructed kidneys significantly increased after UUO. Similar to the intra-renal expression of catalase, the tissue levels of $\mathrm{H}_{2} \mathrm{O}_{2}$ were not different in the obstructed kidneys treated with or without $\mathrm{OA}$ on both day 3 and day 7 (Figure 5A). Oleanolic acid did not lower the renal tissue levels of lipid peroxidation in the UUO-OA mice on day 3. However, it significantly decreased the levels of lipid peroxidation in the obstructed kidneys from UUO-OA mice on day 7 (Figure 5B).

\section{Renal apoptosis}

To investigate whether the nuclear translocation of Nrf2 and the activation of antioxidant enzyme have effects on renal apoptosis, we examined the expression of the proapoptotic protein Bax and the anti-apoptotic protein $\mathrm{Bcl}-2$ by western blot analysis (Figure 6A), together with renal TUNEL-positive cells. UUO increased the Bax expression according to the time course of renal damage.
However, there was no significant difference in the renal Bax expression between UUO-control mice and UUOOA mice. The Bcl-2 expressions were slightly increased after UUO in a time dependent manner. These increases were more prominent after OA treatment on both day 3 and day 7 (Figure 6B).

The number of TUNEL-positive cells increased according to time course of UUO. Oleanolic acid markedly decreased renal TUNEL-positive cells in the obstructed kidneys from UUO-OA mice on both day 3 and day 7 (Figure 1A and 1E).

\section{Discussion}

In the present study, treatment with oleanolic acid attenuated renal inflammation and fibrosis in UUO mice. This study also demonstrated that oleanolic acid suppressed oxidative stress and cellular apoptosis, and promoted antioxidant enzyme expression which may be induced by enhancement of nuclear translocation of cytosolic Nrf2.

Oxidative stress plays a significant role in the progression of tubulointerstitial damage in UUO-induced renal injury [21]. Chronic kidney disease characterized by fibrosis is related to decreased expression of superoxide dismutase and increased expression of NADPH oxidase [22]. UUO also increased lipid peroxidation, shown as the levels of MDA, in the obstructed kidneys, and pretreatment with a superoxide dismutase mimetic reduced the increase in tissue lipid peroxidation after UUO [6]. Excess ROS production from damaged tubular cells or

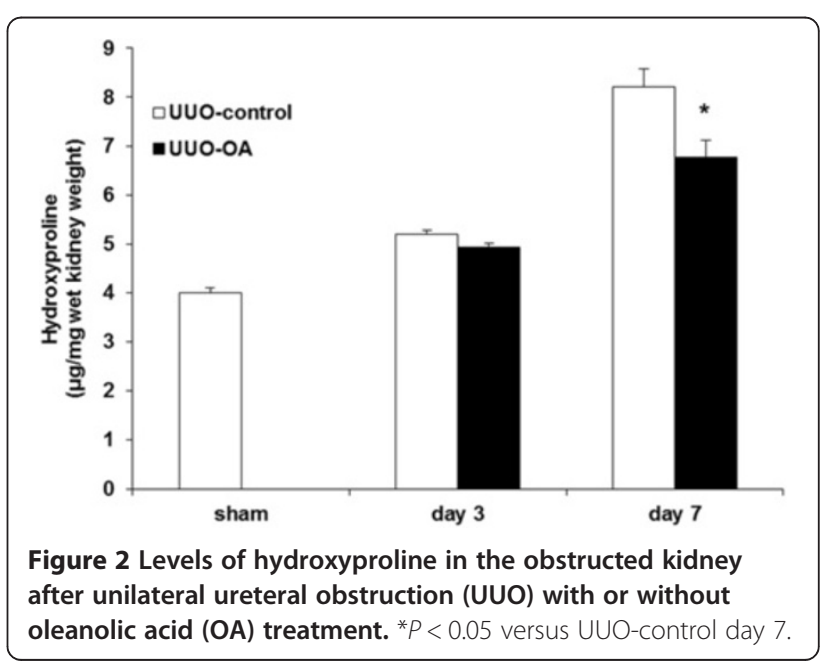



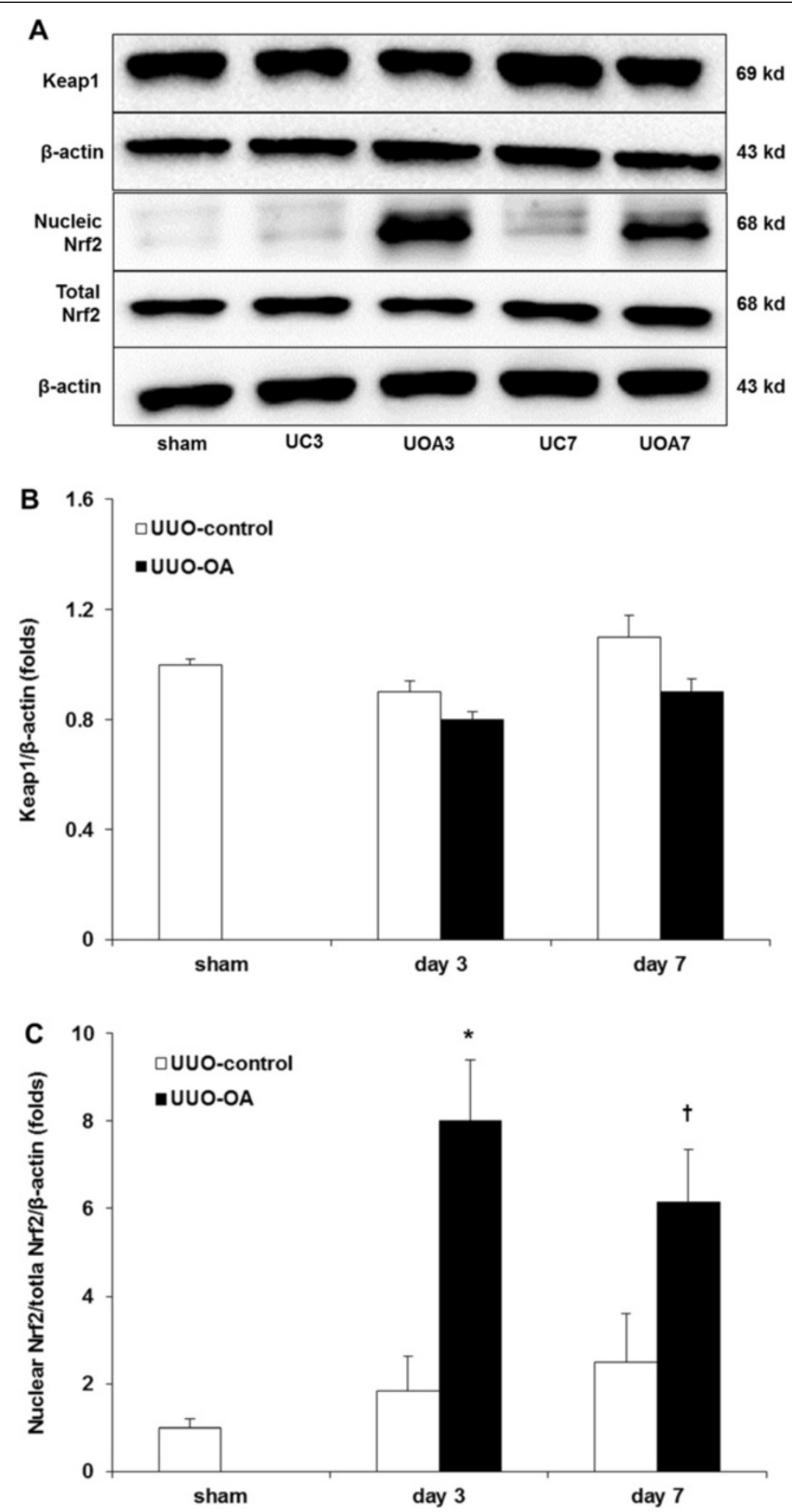

Figure 3 Effects of oleanolic acid (OA) on Keap1 and Nrf2 expression in the obstructed kidney. (A) Representative Western blots of Keap1, total Nrf2 and nuclear Nrf2 in the obstructed kidney after unilateral ureteral obstruction (UUO) with or without OA treatment. (B) The immunofold of the expression of Keap1. (C) The ratio of the expression of nuclear Nrf2 to total Nrf2. UC3, UUO-control day 3; UOA3, UUO-oleanolic acid day 3; UC7, UUO-control day 7; UOA7, UUO-oleanolic acid day $7 ;{ }^{*} P<0.05$ versus UUO-control day 3 group; ${ }^{\dagger} P<0.05$ versus UUO-OA day 7 group. 


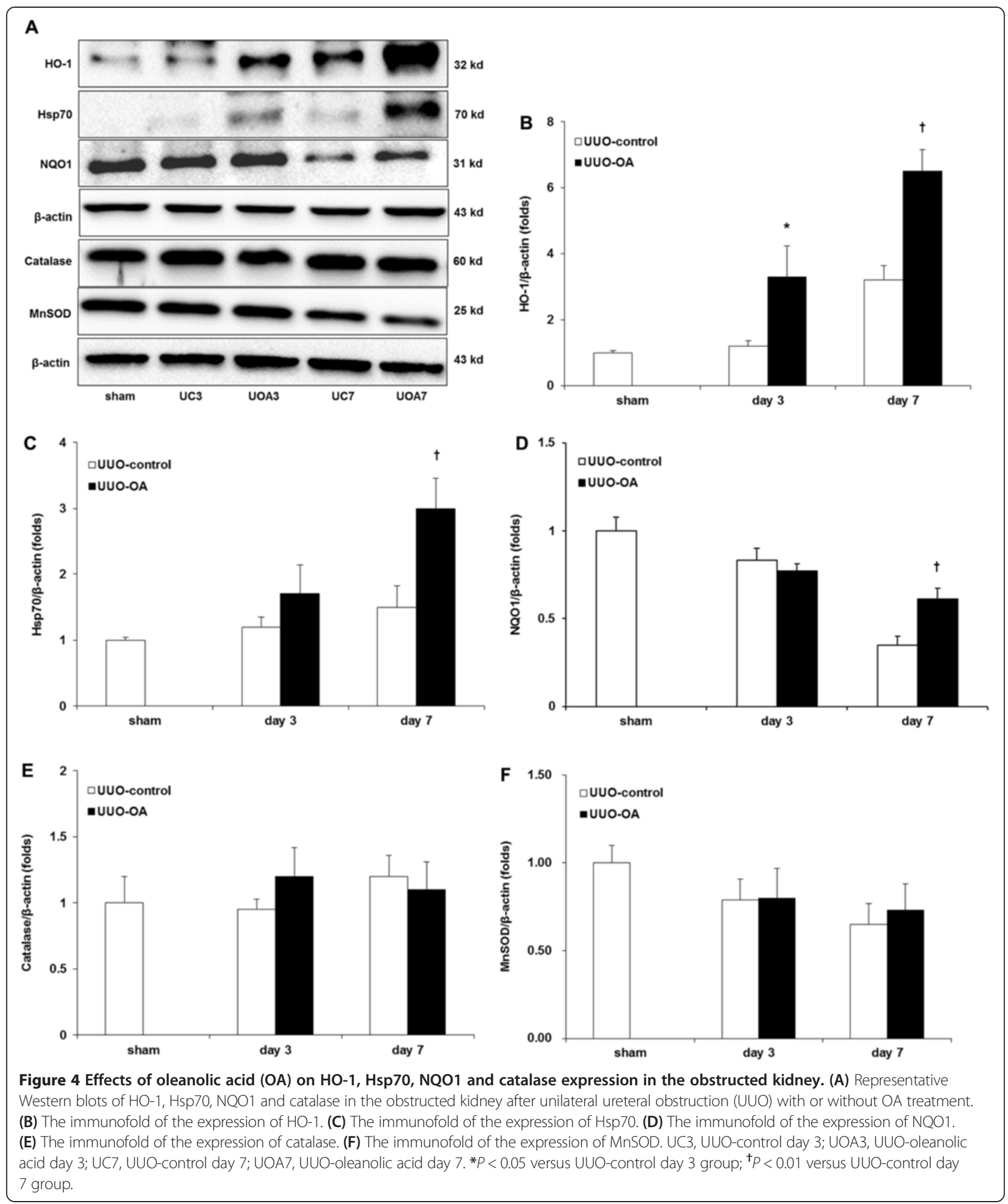

infiltrated leukocytes such as ED-1-positive macrophages leads to renal tubular apoptosis in kidneys after UUO [23]. In the present study, oleanolic acid significantly reduced renal tissue lipid peroxidation and tubular apoptosis, suggesting that oleanolic acid would play a preventative role in the oxidative stress pathway induced by UUO.

Oleanolic acid is a natural triterpenoid that is a constituent of the leaves of Olea europaea, Viscum album $L$. 

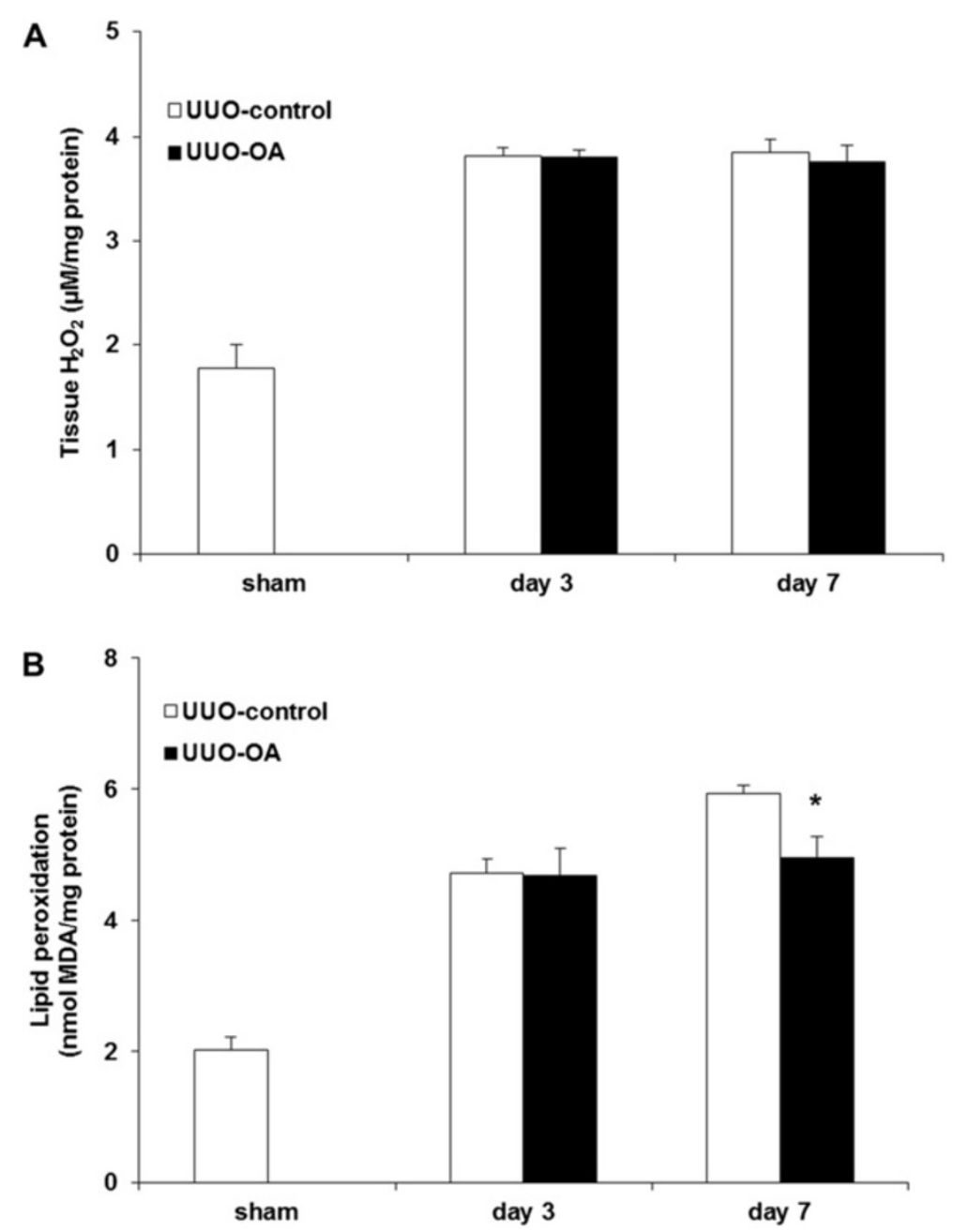

Figure 5 Effect of oleanolic acid $(\mathrm{OA})$ on $\mathrm{H}_{2} \mathrm{O}_{2}$ formation and lipid peroxidation in the obstructed kidney after unilateral ureteral obstruction (UUO). (A) Levels of tissue $\mathrm{H}_{2} \mathrm{O}_{2}$. (B) Levels of lipid peroxidation. ${ }^{*} P<0.05$ versus UUO-control day 7 .

and other plants [8], and has been used as a traditional medicine [24]. It is also found in promace olive oil and is the principal source of fat in the Mediterranean diet, supporting the hypothesis that some components of olive oil may have beneficial effects on human health [9]. Recently, several studies have shown the therapeutic properties of oleanolic acid. One study demonstrated an inhibitory effect of oleanolic acid on the production of advanced glycation end-products in the kidneys of streptozotocininduced diabetic mice [25]. A previous study reported that oleanolic acid improved neurological symptoms in mice with experimental autoimmune encephalomyelitis [9]. The effects of oleanolic acid are thought to result from its anti-inflammatory or anti-oxidant activity [26]. For example, a recent study reported that treatment with oleanolic acid had beneficial effects on the development of atherosclerosis in apolipoprotein E knockout mice, which may be due to its antioxidant properties [11]. Another study demonstrated that oleanolic acid treatment reduced blood pressure in hypertensive animals via endotheliumdependent vasodilatation mediated by nitric oxide [10]. In our study, oleanolic acid reduced total collagen accumulation and interstitial macrophage infiltration in the obstructed kidneys. It also attenuated UUO-induced renal oxidative stress, possibly by up-regulating $\mathrm{HO}-1$, NQO1 and Hsp70 expression and reducing lipid peroxidation. This suggests that oleanolic acid alleviates renal inflammation and fibrosis by decreasing oxidative stress in mice with UUO.

Activation of Nrf2 has been shown to be responsible for oleanolic acid-mediated protection against various insults $[8,15]$. In a recent study, the synthetic triterpenoid analog of oleanolic acid, CDDO-Im, was used in mice fed a highfat diet. Treatment with CDDO-Im prevented high-fat diet-induced obesity in wild-type mice, but not in Nrf2disrupted mice [15]. This finding suggests that oleanolic acid targets Nrf2 signaling. Keap1, a cysteine-rich protein, plays an important role in the inhibition of nuclear 

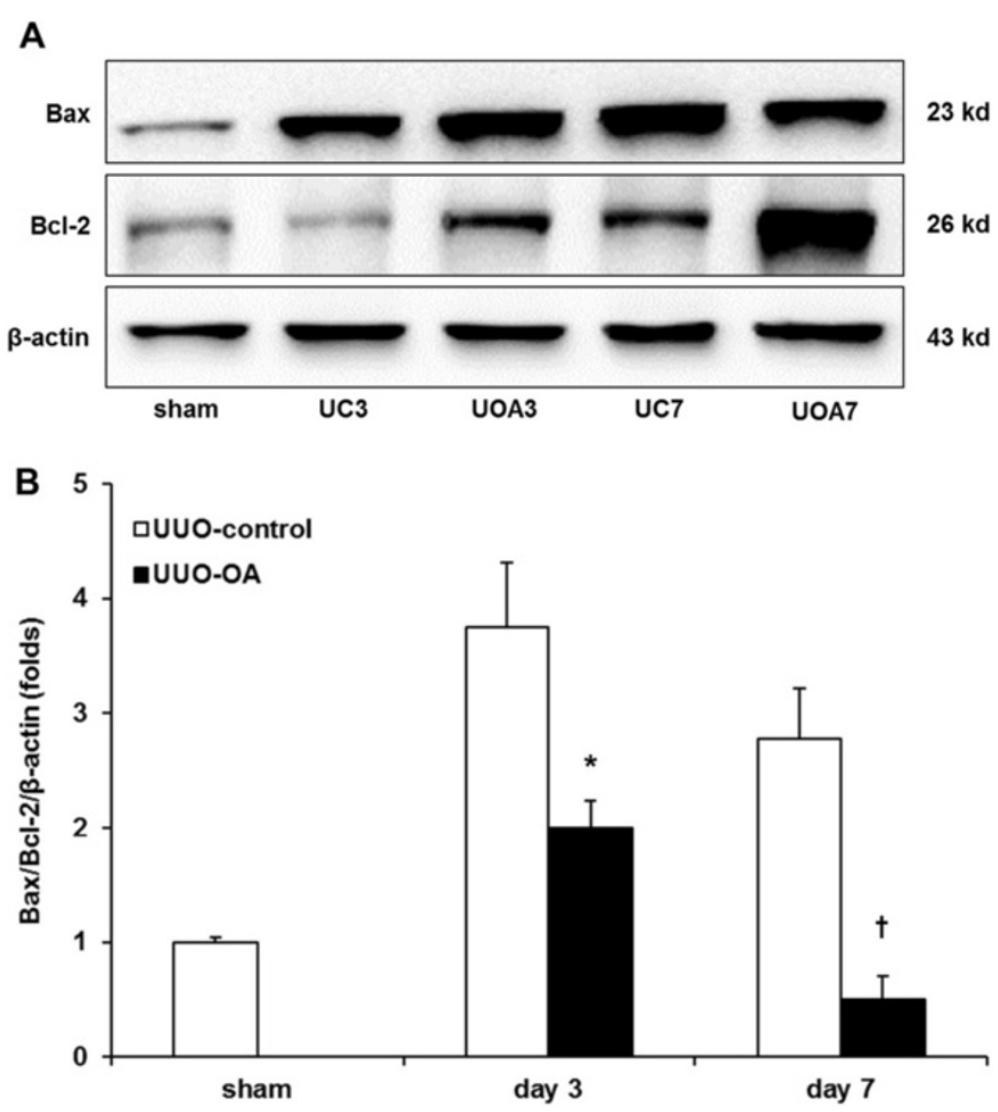

Figure 6 The effects of oleanolic acid (OA) on Bax and Bcl-2 expression in the obstructed kidney. (A) Representative Western blots of Bax and $\mathrm{BCl}-2$ in the obstructed kidney after unilateral ureteral obstruction $(\mathrm{UUO})$ with or without $\mathrm{OA}$ treatment. (B) The ratio of the expression of Bax to BCl-2. UC3, UUO-control day 3; UOA3, UUO-oleanolic acid day 3; UC7, UUO-control day 7; UOA7, UUO-oleanolic acid day 7 . ${ }^{*} P<0.01$ versus UUO-control day 3 group; ${ }^{\dagger} P<0.05$ versus UUO-control day 7 group.

translocation of Nrf2 as well as facilitating proteasomal degradation of Nrf2 [9]. Under normal conditions, Nrf2 is sequestered in the cytoplasm via binding to its repressor molecule, Keap1 [16]. Stressful conditions such as oxidative or ER stress induce the dissociation of the Nrf2Keap1 complex, and subsequent nuclear translocation of Nrf2. This is thought to be the mechanism of Nrf2 activation. An earlier study reported that oleanolic acid facilitated Nrf2 nuclear accumulation, which contributed to protection from acetaminophen hepatotoxicity [8]. Compatible with the study, our results showed that oleanolic acid enhanced the expression of nuclear Nrf2, resulting in an increased nuclear Nrf2/total Nrf2 ratio in the obstructed kidneys. In the present study, UUO or oleanolic acid treatment did not change the renal expression of Keap1 and total Nrf2 in the obstructed kidneys. On the other hand, a previous study demonstrated a paradoxical reduction of inactivated Nrf2, accompanied by a significant elevation of Keap1 in cases of severe oxidative stress or inflammation [16]. Although the reason for this discrepancy remains unclear, the changes in Keap1 and total Nrf2 levels may differ according to experimental models of oxidative stress and the severity of renal injury. Taken together, our results may suggest that oleanolic acid facilitates nuclear translocation of cytosolic Nrf2 rather than directly inducing dissociation of the Nrf2-Keap1 complex.

Within the nucleus, Nrf2 binds to regulatory sequences called antioxidant response elements or electrophile response elements, which are located in the promoter region of genes encoding the antioxidant and phase 2 detoxifying enzymes such as glutathione-S-transferases, NQO1, glutamate-cysteine ligase catalytic subunit, catalase, thioredoxin, and HO-1 $[8,16]$. The present study showed that oleanolic acid markedly increased the expression of HO-1, an important antioxidant enzyme, in the obstructed kidneys of oleanolic acid-treated mice. In general, HO-1 metabolizes heme that accumulates in tissues as a by-product of red blood cell turnover. Metabolites produced by such degradation reactions have been known to trigger signaling cascades that aid antioxidant defenses and protect against pathological increases in oxidative stress [27]. Unlike $\mathrm{HO}-1$, in our results, expression of NQO1 upregulated by oleanolic acid was more prominent on day 7 than on day 3. There appears to be some 
differences in the expression of phase 2 enzymes according to the experimental model: the regulatory mechanisms of phase 2 enzymes in relation to the Nrf2 pathway may be tissue- or cell-specific [28]. Our results suggest that $\mathrm{HO}-1$, rather than $\mathrm{NQO} 1$, plays a major role in the attenuation of renal inflammation and fibrosis in this UUO model. We also observed that oleanolic acid treatment increased Hsp70 protein expression in renal tissue on UUO day 7. This finding accords with previous results that activation of $\mathrm{Nrf} 2$ upregulates its downstream gene products and the Hsp70 gene [29]. Our results demonstrated that oleanolic acid improved interstitial fibrosis and the total collagen content of renal tissue on day 7 after UUO, although attenuation of tubular injury and interstitial inflammation by oleanolic acid was evident even in day 3. Considering that renal fibrosis in our study was further attenuated on day 7 than it was on day 3, late upregulation of NQO1 and Hsp70 might have synergistic effects on the suppression of progressive fibrosis resulting from UUO. On the other hand, there was no difference in the expression of catalase, which is responsible for $\mathrm{H}_{2} \mathrm{O}_{2}$ neutralization, in obstructed kidneys with or without OA treatment. It is unclear why oleanolic acid did not change the expression of catalase together with the levels of tissue $\mathrm{H}_{2} \mathrm{O}_{2}$ in this study. In addition, MnSOD protein level showed no differences with administration of oleanolic acid. Taking findings of previous studies into consideration $[6,12]$, we cautiously speculate that the type of oxidative stress may influence the detailed action of Nrf2 that regulates induction of genes encoding specific antioxidants.

The present study demonstrated that renal apoptosis was attenuated by treatment with oleanolic acid. This finding is consistent with a recent study showed that the up-regulation of HO-1 significantly decreased cellular apoptosis [30]. Our study also showed that although oleanolic acid had no effect on the levels of the proapoptotic enzyme Bax it increased the expression of the anti-apoptotic enzyme $\mathrm{Bcl}-2$, resulting in a decreased $\mathrm{Bax} / \mathrm{Bcl}-2$ ratio. It has been suggested that Nrf2 targets the anti-apoptotic $\mathrm{Bcl}-2$ protein, and that antioxidant treatment releases $\mathrm{Nrf} 2$ and increases $\mathrm{Bcl}-\mathrm{xL}$ heterodimerization with Bax, resulting in reduced cellular apoptosis [31]. Our results suggest that $\mathrm{HO}-1$ and $\mathrm{Bcl}-2$ may serve as key players in Nrf2-upregulated cell survival under oxidative stress induced by UUO. Furthermore, the interaction between $\mathrm{Hsp} 70$ and $\mathrm{Bcl}-2$ is considered to contribute to renoprotection in this study. It has been known that Hsp70 can inhibit apoptosis by antagonizing the apoptosis-inducing factor [32].

The current study has some limitations. First, we did not measure the blood urea nitrogen (BUN) and serum creatinine as renal functional parameters. However, many previous studies have reported that BUN or serum creatinine was not significantly affected by UUO because of the presence of a contralateral kidney with good renal function [33,34], suggesting that BUN and serum creatinine are not good indicators of renal function in an animal model of UUO. Second, we could not evaluate the molecular mechanism through which oleanolic acid modulates nuclear translocation of Nrf2. Third, we did not investigate the possible Nrf2-independent mechanisms that could contribute to renoprotection in the UUO model. Like a previous report [8], we found that oleanolic acid treatment increased the expression of Hsp70 protein that may contribute to Nrf2-independent renoprotection. A previous study used microarray to compare the overall gene expression signatures modulated by pharmacologic or genetic activation of Nrf2 signaling in liver tissue and found several Keap1-Nrf2-independent genes [35]. Another report indicated that oleanolic acid protected against hepatotoxicity in wild type mice, but did so to a lesser degree in Nrf2-null mice, suggesting that oleanolic acid could activate Nrf2-independent protective mechanisms [8]. Further investigation is needed to elucidate the exact mechanisms of the Nrf2-independent effects of oleanolic acid.

\section{Conclusions}

In conclusion, we found that oleanolic acid treatment protects against oxidative insults in obstructed kidneys after UUO. Oleanolic acid appears to exert its beneficial effects by facilitating nuclear translocation of Nrf2 and subsequently up-regulating antioxidant and anti-apoptotic enzymes. Treatment with oleanolic acid may provide a therapeutic approach in preventing renal oxidative stress, inflammation, and fibrosis.

\section{Competing interests}

The authors declare that there are no competing interests.

\section{Authors' contributions}

The experiment was designed and implemented by SC and SJS. Data were analyzed by HEY, SJK, SJK, ESK, YAH, CWP, YSC and SJS. SC and SJS prepared the manuscript. SC and SJS supervised overall project. All authors read and approved the final version of manuscript.

\section{Acknowledgements}

This research was supported by Basic Science Research Program through the National Research Foundation of Korea (NRF) funded by the Ministry of Education, Science and Technology (20110013312), the financial support of the Catholic Medical Center Research Foundation made in the program year of 2011 (52011B000100001), and the Incheon St. Mary's Hospital of the Catholic University of Korea, through Clinical Research Laboratory Foundation Program, 2012 (52012B000100138). We thank Dr. Allison A. Eddy in University of Washington for methodological help with measurement of total collagen, and thank Dr. Kwon Moo Park in Kyungpook National University for methodological help with measurement of lipid peroxidation and levels of tissue $\mathrm{H}_{2} \mathrm{O}_{2}$

\section{Author details}

'Department of Internal Medicine, College of Medicine, The Catholic University of Korea, 222 Banpo-daero, Seoul 137-701, Republic of Korea. 2Division of Nephrology, The Catholic University of Korea Yeouido St. Mary's Hospital, 10, 63-ro, Yeongdeungpo-gu, Seoul 150-713, Republic of Korea. 
${ }^{3}$ Division of Nephrology, The Catholic University of Korea Incheon St. Mary's Hospital, 56, Dongsu-ro, Bupyeong-gu, Incheon 403-720, Republic of Korea. ${ }^{4}$ Division of Nephrology, The Catholic University of Korea Seoul St. Mary's Hospital, 222, Banpo-daero, Seoul 137-701, Republic of Korea. ${ }^{5}$ Division of Nephrology, Korea University Guro Hospital, 148, Gurodong-ro, Guro-gu, Seoul 152-703, Republic of Korea.

Received: 22 August 2013 Accepted: 1 January 2014

Published: 6 January 2014

\section{References}

1. Chatziantoniou C, Dussaule JC: Insights into the mechanisms of renal fibrosis: is it possible to achieve regression? Am J Physiol Renal Physiol 2005, 289:F227-F234

2. Jin DC, Ha IS, Kim NH, Lee SW, Lee JS, Yoon SR, Kim BS: Brief Report: renal replacement therapy in Korea, 2010. Kidney Res Clin Pract 2012, 31:62-71.

3. Cho MH, Jung KJ, Jang HS, Kim JI, Park KM: Orchiectomy attenuates kidney fibrosis after ureteral obstruction by reduction of oxidative stress in mice. Am J Nephrol 2012, 35:7-16.

4. Manucha W: Biochemical-molecular markers in unilateral ureteral obstruction. Biocell 2007, 31:1-12.

5. Ozbek E, llbey YO, Ozbek M, Simsek A, Cekmen M, Somay A: Melatonin attenuates unilateral ureteral obstruction-induced renal injury by reducing oxidative stress, iNOS, MAPK, and NF-kB expression. J Endourol 2009, 23:1165-1173.

6. Dendooven A, Ishola DA Jr, Nguyen TQ, Van der Giezen DM, Kok RJ Goldschmeding R, Joles JA: Oxidative stress in obstructive nephropathy. Int J Exp Pathol 2011, 92:202-210.

7. Truong LD, Gaber L, Eknoyan G: Obstructive uropathy. Contrib Nephrol 2011, 169:311-326.

8. Reisman SA, Aleksunes LM, Klaassen CD: Oleanolic acid activates Nrf2 and protects from acetaminophen hepatotoxicity via Nrf2-dependent and Nrf2-independent processes. Biochem Pharmacol 2009, 77:1273-1282.

9. Martín R, Carvalho-Tavares J, Hernández M, Arnés M, Ruiz-Gutiérrez V, Nieto ML: Beneficial actions of oleanolic acid in an experimental model of multiple sclerosis: a potential therapeutic role. Biochem Pharmacol 2010, 79:198-208.

10. Bachhav SS, Patil SD, Bhutada MS, Surana SJ: Oleanolic acid prevents glucocorticoid-induced hypertension in rats. Phytother Res 2011, 25:1435-1439.

11. Buus NH, Hansson NC, Rodriquez-Rodriguez R, Stankevicius E, Andersen MR, Simonsen U: Antiatherogenic effects of oleanolic acid in apolipoprotein $\mathrm{E}$ knockout mice. Eur J Pharmacol 2011, 670:519-526.

12. Santos RS, Silva PL, Oliveira GP, Cruz FF, Ornellas DS, Morales MM, Fernandes J, Lanzetti M, Valença SS, Pelosi P, Gattass CR, Rocco PR: Effects of oleanolic acid on pulmonary morphofunctional and biochemical variables in experimental acute lung injury. Respir Physiol Neurobiol 2011, 179:129-136.

13. Liu J, Liu Y, Klaassen CD: The effect of Chinese hepatoprotective medicines on experimental liver injury in mice. J Ethnopharmacol 1994, 42:183-191.

14. Liu Y, Kreppel H, Liu J, Choudhuri S, Klaassen CD: Oleanolic acid protects against cadmium hepatotoxicity by inducing metallothionein. $J$ Pharmacol Exp Ther 1993, 266:400-406.

15. Shin S, Wakabayashi J, Yates MS, Wakabayashi N, Dolan PM, Aja S, Liby KT, Sporn MB, Yamamoto M, Kensler TW: Role of Nrf2 in prevention of highfat diet-induced obesity by synthetic triterpenoid CDDO-imidazolide. Eur J Pharmacol 2009, 620:138-144

16. Kim HJ, Vaziri ND: Contribution of impaired Nrf2-Keap1 pathway to oxidative stress and inflammation in chronic renal failure. Am J Physiol Renal Physiol 2010, 298:F662-F671.

17. Zhang G, Kim H, Cai X, López-Guisa JM, Alpers CE, Liu Y, Carmeliet P, Eddy AA: Urokinase receptor deficiency accelerates renal fibrosis in obstructive nephropathy. J Am Soc Nephrol 2003, 14:1254-1271.

18. Matsuo S, López-Guisa JM, Cai X, Okamura DM, Alpers CE, Bumgarner RE, Peters MA, Zhang G, Eddy AA: Multifunctionality of PAl-1 in fibrogenesis: evidence from obstructive nephropathy in PAl-1-overexpressing mice. Kidney Int 2005, 67:2221-2238.

19. Radford MG Jr, Donadio JV Jr, Bergstralh EJ, Grande JP: Predicting renal outcome in IgA nephropathy. J Am Soc Nephrol 1997, 8:199-207.
20. Kim J, Kil IS, Seok YM, Yang ES, Kim DK, Lim DG, Park JW, Bonventre JV, Park KM: Orchiectomy attenuates post-ischemic oxidative stress and ischemia/ reperfusion injury in mice. A role for manganese superoxide dismutase. $J$ Biol Chem 2006, 281:20349-20356.

21. Kramer AB, van der Meulen EF, Hamming I, Van Goor H, Navis G: Effect of combining ACE inhibition with aldosterone blockade on proteinuria and renal damage in experimental nephrosis. Kidney Int 2007, 71:417-424.

22. Vaziri ND, Dicus M, Ho ND, Boroujerdi-Rad L, Sindhu RK: Oxidative stress and dysregulation of superoxide dismutase and NADPH oxidase in renal insufficiency. Kidney Int 2003, 63:179-185.

23. Chen CF, Yeh SU, Chien CT, Wu MS: Renal response during acute unilateral ureteral obstruction in rats. Neurourol Urodyn 2001, 20:125-137.

24. Ji HY, Shin BS, Jeong DW, Park EJ, Park ES, Yoo SD, Lee HS: Interspecies scaling of oleanolic acid in mice, rats, rabbits and dogs and prediction of human pharmacokinetics. Arch Pharm Res 2009, 32:251-257.

25. Wang ZH, Hsu CC, Huang CN, Yin MC: Anti-glycative effects of oleanolic acid and ursolic acid in kidney of diabetic mice. Eur J Pharmacol 2010, 628:255-260.

26. Nataraju A, Saini D, Ramachandran S, Benshoff N, Liu W, Chapman W, Mohanakumar T: Oleanolic Acid, a plant triterpenoid, significantly improves survival and function of islet allograft. Transplantation 2009, 88:987-994.

27. Haines DD, Lekli I, Teissier P, Bak I, Tosaki A: Role of haeme oxygenase-1 in resolution of oxidative stress-related pathologies: focus on cardiovascular, lung, neurological and kidney disorders. Acta Physiol (Oxf) 2012, 204:487-501.

28. Piao MS, Park JJ, Choi JY, Lee DH, Yun SJ, Lee JB, Lee SC: Nrf2-dependent and Nrf2-independent induction of phase 2 detoxifying and antioxidant enzymes during keratinocyte differentiation. Arch Dermatol Res 2012, 304:387-395.

29. Manucha W, Kurbán F, Mazzei L, Benardón ME, Bocanegra V, Tosi MR, Vallés $P:$ eNOS/Hsp70 interaction on rosuvastatin cytoprotective effect in neonatal obstructive nephropathy. Eur J Pharmacol 2011, 650:487-495.

30. Banerjee P, Basu A, Datta D, Gasser M, Waaga-Gasser AM, Pal S: The heme oxygenase-1 protein is overexpressed in human renal cancer cells following activation of the Ras-Raf-ERK pathway and mediates antiapoptotic signal. J Biol Chem 2011, 286:33580-33590.

31. Niture SK, Jaiswal AK: Inhibitor of Nrf2 (INrf2 or Keap1) protein degrades $\mathrm{BCl}-\mathrm{xL}$ via phosphoglycerate mutase 5 and controls cellular apoptosis. J Biol Chem 2011, 286:44542-44556.

32. Arambašić J, Mihailović M, Uskoković A, Dinić S, Grdović N, Marković J, Poznanović G, Bajec D, Vidaković M: Alpha-lipoic acid upregulates antioxidant enzyme gene expression and enzymatic activity in diabetic rat kidneys through an O-GlcNAc-dependent mechanism. Eur J Nutr 2013, 52:1461-1473

33. Ning XH, Ge XF, Cui Y, An HX: Ulinastatin inhibits unilateral ureteral obstruction-induced renal interstitial fibrosis in rats via transforming growth factor $\beta$ (TGF- $\beta$ )/Smad signalling pathways. Int Immunopharmacol 2013, 15:406-413.

34. Wu WP, Chang CH, Chiu YT, Ku CL, Wen MC, Shu KH, Wu MJ: A reduction of unilateral ureteral obstruction-induced renal fibrosis by a therapy combining valsartan with aliskiren. Am J Physiol Renal Physiol 2010, 299:F929-F941.

35. Yates MS, Tran QT, Dolan PM, Osburn WO, Shin S, McCulloch CC, Silkworth JB, Taguchi K, Yamamoto M, Williams CR, Liby KT, Sporn MB, Sutter TR, Kensler TW: Genetic versus chemoprotective activation of Nrf2 signaling: overlapping yet distinct gene expression profiles between Keap1 knockout and triterpenoid-treated mice. Carcinogenesis 2009, 30:1024-1031.

doi:10.1186/1743-7075-11-2

Cite this article as: Chung et al: Oleanolic acid attenuates renal fibrosis in mice with unilateral ureteral obstruction via facilitating nuclear translocation of Nrf2. Nutrition \& Metabolism 2014 11:2. 